\title{
Comparison of Vandenbos procedure or Winograd method for ingrown toenail
}

\author{
Erkan Karacan, $\mathrm{MD}^{1}{ }^{1}$, Devran Ertilav, $\mathrm{MD}^{2}$ (1) \\ 'Department of General Surgery, Aydın State Hospital, Aydın, Turkey \\ ${ }^{2}$ Department of Orthopedics and Traumatology, Aydın State Hospital, Aydın, Turkey
}

Ingrown toenail is a painful, irritating nail disorder that causes inflammation, infection, and the formation of excessive granulation tissue. ${ }^{[1]}$ An ingrown toenail is a common condition which negatively affects daily life due to pain. Although it can occur at any age, it usually affects young individuals..$^{[2]}$

In 1959, Vandenbos and Bowers ${ }^{[3]}$ suggested that, rather than the nail, the soft tissue surrounding the nail grew due to pressure during daily activities, leading to pressure necrosis in the tissue around the nail, which contributes significantly to the pathogenesis of onychocryptosis. Vandenbos ${ }^{[3]}$ considered that the most important factor in onychocryptosis was the overgrown toe skin and he developed a surgical procedure. In this procedure, only overgrown soft tissue is removed without matricectomy and damaging the nail. This method is considered a radical intervention; therefore, it has not been commonly to date.

Received: January 13, 2021

Accepted: April 07, 2021

Published online: June 11, 2021

Correspondence: Erkan Karacan, MD. Aydın Devlet Hastanesi Genel Cerrahi Kliniği, 09100 Efeler, Aydın, Türkiye.

E-mail: erkan8806@gmail.com

Doi: $10.52312 /$ jdrs.2021.36

Citation: Karacan E, Ertilav D. Comparison of Vandenbos procedure or Winograd method for ingrown toenail. Jt Dis Relat Surg 2021;32(2):414-419.

(C2021 All right reserved by the Turkish Joint Diseases Foundation

This is an open access article under the terms of the Creative Commons Attribution-NonCommercial License, which permits use, distribution and reproduction in any medium, provided the original work is properly cited and is not used for commercial purposes (http://creativecommons.org/licenses/by-nc/4.0/).

\section{ABSTRACT}

Objectives: This study aims to compare the outcomes of the Winograd method and Vandenbos procedure used to treat an ingrown toenail.

Patients and methods: Between January 2017 and February 2020, a total of 145 patients (65 males, 80 females; mean age: 31.45 years; range, 13 to 61 years) who were treated with Winograd or Vandenbos procedure for an ingrown toenail were retrospectively analyzed. Of the patients, 70 underwent the Winograd method (Group 1) and 75 underwent the Vandenbos procedure (Group 2). Postoperative Visual Analog Scale (VAS) scores, demographic data, recurrence/complication rates, and satisfaction of patients were recorded.

Results: The mean recovery time was $11.8 \pm 2.6$ days in Group 1 and $18.0 \pm 2.1$ days in Group $2(\mathrm{p}<0.001)$. The mean VAS score was $3.9 \pm 0.7$ in Group 1 and $7.2 \pm 1.0$ in Group $2(\mathrm{p}<0.001)$ during the first postoperative week. Of the patients, $56(80 \%)$ of 70 patients in Group 1 and 74 (98\%) of 75 patients in Group 2 were satisfied with the surgery $(\mathrm{p}<0.001)$. In terms of cosmetics, $60(85 \%)$ of the patients in Group 1 and $74(98 \%)$ of the patients in Group 2 were satisfied ( $\mathrm{p}=0.003$ ). Recurrence was observed in $10(14 \%)$ patients in Group 1, while no recurrence was observed in Group 2. Six (8.7\%) patients in Group 1 and one (1.3\%) patient in Group 2 developed complications.

Conclusion: Low recurrence rates, high satisfaction, and good cosmetic results can be achieved with the Vandenbos procedure, while recovery time and return to work are faster with the Winograd method. The appropriate surgical technique should be selected based on the individual patient.

Keywords: Ingrown toenail, Vandenbos procedure, Winograd method.

However, successful results have been reported in the literature. ${ }^{[4]}$

The Winograd method was first described by Winograd, ${ }^{[5]}$ an American podiatrist, in 1929. In this technique, wedge resection and matricectomy were used. ${ }^{[6]}$ However, despite the frequent use of this technique, there are publications reporting recurrence and poor cosmetic results. ${ }^{[7]}$ 
There are very few reports in the literature regarding the comparison of surgical treatments for ingrown toenails. ${ }^{[8]}$ Therefore, in this study, we aimed to compare the recurrence, pain, recovery time, time to return to work, cosmetic results, and postoperative complication rates of the frequently preferred Winograd method and the less frequently preferred Vandenbos procedure.

\section{PATIENTS AND METHODS}

This single-center, retrospective study was conducted at Aydin State Hospital, Departments of General Surgery and Orthopedics and Traumatology between January 2017 and February 2020. The files of the patients who underwent the Vandenbos procedure and those who underwent the Winograd method were reviewed. Only patients who were under follow-up for at least six months after surgery were included in the study. Patients who underwent the modified Vandenbos procedure and who were under follow-up for less than six months were excluded from the study. Finally, a total of 145 patients (65 males, 80 females; mean age: 31.45 years; range, 13 to 61 years) were included. Of the patients, 70 underwent the Winograd method (Group 1) and 75 underwent the Vandenbos procedure (Group 2). A written informed consent was obtained from each patient. The study protocol was approved by the Adnan Menderes University Faculty of Medicine Ethics Committee (No: 2020/158). The study was conducted in accordance with the principles of the Declaration of Helsinki.

The patients included in the study were grouped according to the type of surgery. The demographic data of the patients were reviewed retrospectively and recorded. The postoperative one-week, one-month, three-month, and six-month outpatient follow-up notes were evaluated. In the outpatient follow-ups, their recovery time and Visual Analog Scale (VAS) scores were evaluated. We evaluated the recovery time as the time of wearing shoes. The satisfaction levels of the patients were indicated with "Yes/No" according to the outpatient clinic notes. Cosmetic satisfaction was indicated with "Yes/No" according to clinic notes. The patients were also evaluated for recurrence and complications.

\section{Surgical technique}

For the Vandenbos procedure, the toe was cleaned with iodine solution, and a digital nerve block without epinephrine was performed with $2 \%$ prilocaine. An elastic tourniquet was placed proximal to the toe to provide a bloodless operating field and administration of local anesthetic into the tissue. An incision, measuring $5 \mathrm{~mm}$ in size, was made proximally while leaving the nail bed intact. Beginning approximately $3 \mathrm{~mm}$ from the lateral edge of the base of the nail, the nail matrix was protected. An elliptical sweep was made out to the side and bottom of the toe, extending distally to encompass all involved granulation tissue and nail-fold skin. The incision continued approximately 3 to $5 \mathrm{~mm}$ from the edge below the nail tip. The skin and subcutaneous tissues at the edge of the nail were removed. The excision should be large and sufficient. If enough tissue was removed, skin and soft-tissue defect, measuring $1.5 \times 3 \mathrm{~cm}$, was able to be formed. The tourniquet was opened and pressure was applied for $3 \mathrm{~min}$, bleeding foci were cauterized to reduce postoperative bleeding. At this stage, a special care was paid not to damage the nail matrix. The wound should be left to secondary tissue healing. There would be bleeding due to postoperative leakage, a large amount of non-stick gauze should be applied to the wound area with pressure.

\section{Statistical analysis}

Statistical analysis was performed using the IBM SPPS for Windows version 25.0 software (IBM Corp., Armonk, NY, USA). Descriptive data were expressed in mean \pm standard deviation (SD), median (min-max) or number and frequency, where applicable. The Kolmogorov-Smirnov test was performed to evaluate the homogeneity of the data. The Student t-test was used for the comparison of two groups. When the Student t-test did not provide preconditions, the Mann-Whitney $U$ test was used. Categorical data were analyzed using the Fisher's exact test and chi-square test. A $p$ value of $<0.05$ was considered statistically significant.

\section{RESULTS}

Of the patients, the mean age was $31.8 \pm 12.7$ (range, 13 to 58) years in Group 1 and $31.2 \pm 13.1$ (range, 13 to 61) years in Group 2. The mean recovery time was $11.8 \pm 2.6$ days in Group 1 and $18.0 \pm 2.1$ days in Group 2 ( $\mathrm{p}<0.001)$.

The mean VAS score during the first postoperative week was $3.9 \pm 0.7$ in Group 1 and $7.2 \pm 1.0$ in Group $2(\mathrm{p}<0.001)$. The mean VAS score during the first postoperative month was $1.8 \pm 0.7$ in Group 1 and $1.6 \pm 0.7$ in Group 2 ( $p=0.06)$. The mean VAS score during the third postoperative month was $1.3 \pm 0.4$ in Group 1 and $0.3 \pm 0.5$ in Group 2 ( $p<0.001$ ). The mean VAS score during the postoperative sixth month was $0.4 \pm 1.0$ in Group 1 and $0.1 \pm 0.5$ in Group 2 $(\mathrm{p}<0.001)$. 


\begin{tabular}{|c|c|c|c|c|c|c|c|}
\hline \multicolumn{8}{|c|}{ TABLE I } \\
\hline & \multicolumn{3}{|c|}{ Group $1(n=70)$} & \multicolumn{3}{|c|}{ Group $2(\mathrm{n}=75)$} & \multirow[b]{2}{*}{$p$} \\
\hline & $\mathrm{n}$ & $\%$ & Mean $\pm S D$ & $\mathrm{n}$ & $\%$ & Mean $\pm S D$ & \\
\hline Age (year) & & & $31.8 \pm 12.7$ & & & $31.2 \pm 13.1$ & 0.77 \\
\hline Recovery time (day) & & & $11.8 \pm 2.6$ & & & $18.0 \pm 2.1$ & $<0.001$ \\
\hline Preoperative VAS & & & $8.1 \pm 0.7$ & & & $8.2 \pm 0.8$ & 0.24 \\
\hline VAS in postoperative first week & & & $3.9 \pm 0.7$ & & & $7.2 \pm 1.0$ & $<0.001$ \\
\hline VAS in postoperative first month & & & $1.8 \pm 0.7$ & & & $1.6 \pm 0.7$ & 0.06 \\
\hline VAS in postoperative third month & & & $1.3 \pm 0.4$ & & & $0.3 \pm 0.5$ & $<0.001$ \\
\hline VAS in postoperative sixth month & & & $0.4 \pm 1.0$ & & & $0.1 \pm 0.5$ & $<0.001$ \\
\hline Satisfaction & & 80 & & & 98 & & $<0.001$ \\
\hline Cosmetic satisfaction & & 85 & & & 98 & & 0.003 \\
\hline Recurrence & 10 & & & 0 & & & $<0.001$ \\
\hline Complication & 6 & & & 1 & & & 0.056 \\
\hline Bleeding & 0 & & & 1 & 2.66 & & \\
\hline Infection & 6 & 8.57 & & 0 & & & \\
\hline
\end{tabular}

While $56(80 \%)$ of the 70 patients who underwent Winograd surgery satisfied with the surgery, $74(98 \%)$ of the 75 patients who underwent Vandenbos procedure were satisfied with the surgery $(p<0.001)$. When the patients were evaluated in terms of cosmetics, $60(85 \%)$ of the patients in Group 1 and $74(98 \%)$ of the patients in Group 2 were satisfied $(p=0.003)$. The pre- and postoperative images of the
Vandenbos procedure and Winograd method are shown in Figures 1 and 2.

Recurrence was observed in $10(14 \%)$ patients in Group 1, while none of the patients in Group 2 experienced recurrence $(\mathrm{p}<0.001)$.

Six $(8.7 \%)$ patients in Group 1 and one $(1.3 \%)$ patient in Group 2 developed complications $(\mathrm{p}=0.056)$.

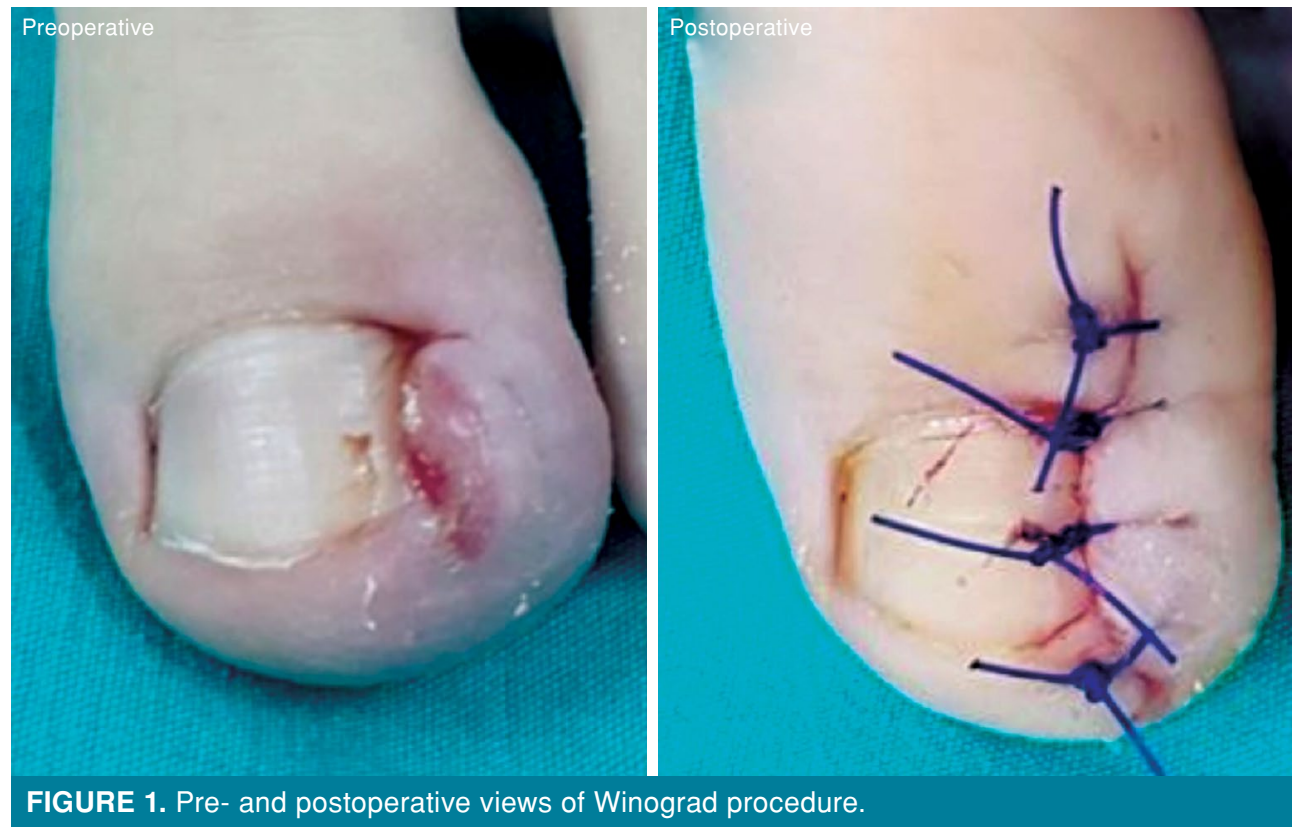



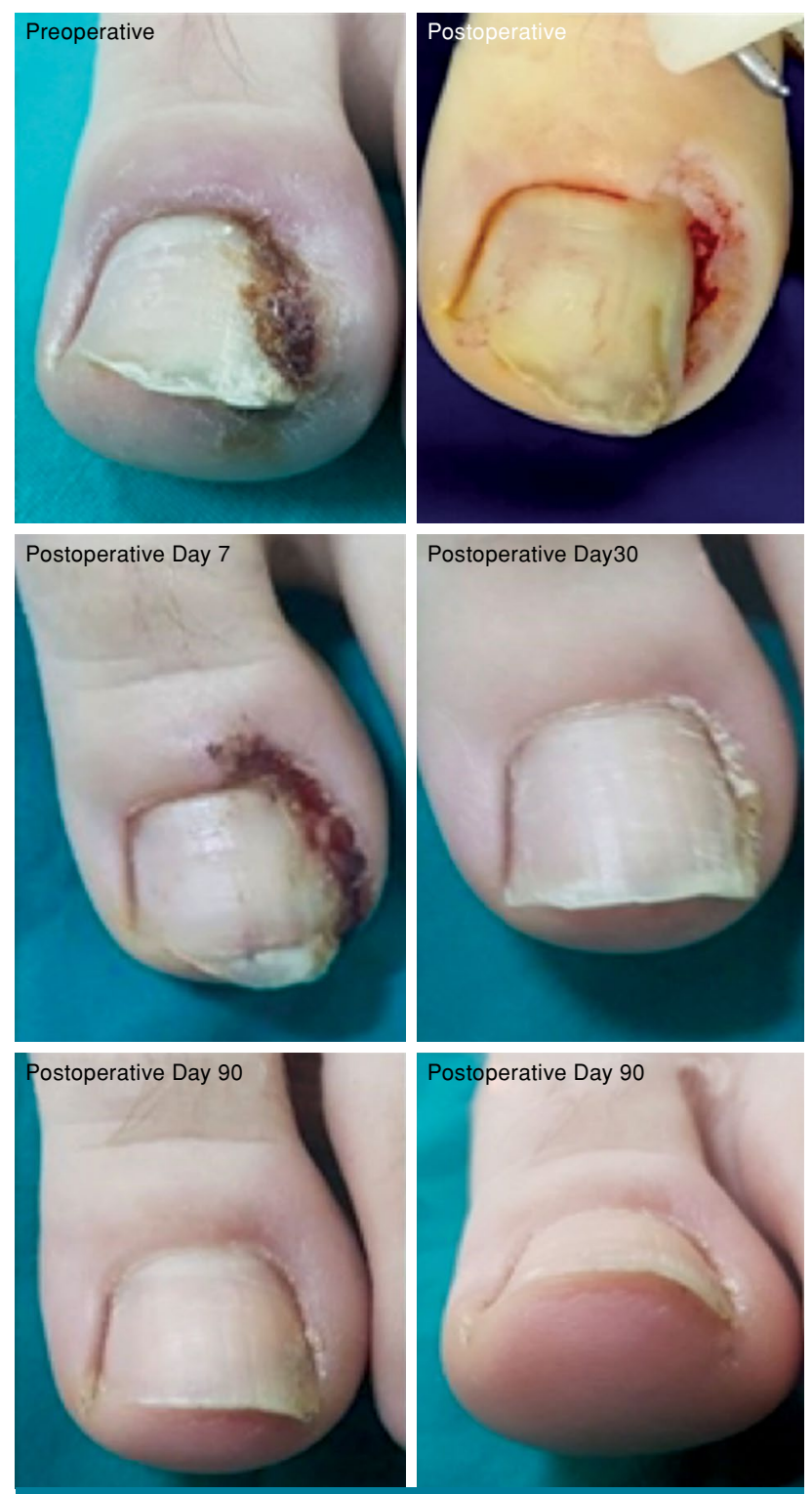

FIGURE 2. Pre- and postoperative views of Vandenbos procedure.

The comparative results of the Winograd and Vandenbos procedures are summarized in Table I.

\section{DISCUSSION}

The most important finding of this study was that Vandenbos procedure has low recurrence and a higher cosmetic satisfaction rate. ${ }^{[9]}$ Although ingrown toenail is a condition that can occur at any age, it commonly affects younger individuals and restricts the daily physical activities of the patient, and therefore, it must be treated. Currently, no ideal surgical technique has been defined for the treatment of ingrown toenails. The characteristics of an ideal treatment for ingrown toenail are that postoperative comfort should be good, it should provide a quick return to normal activities, and should be simple and inexpensive. ${ }^{[8]}$ Moreover, acceptable cosmetic results, as well as low complication and recurrence rates, are of importance. ${ }^{[7]}$

Although the Winograd method is widely used for ingrown toenail surgery, its recurrence rates vary in studies. For instance, a study reported the recurrence rate as $16 \%$ following wedge resection and partial matricectomy. ${ }^{[10]}$ Moreover, two different comparative studies reported the recurrence rates as $21 \%$ and $7 \%$, respectively. ${ }^{[7,11]}$ In a study comparing the Winograd method and sleeve technique, the recurrence rate of the Winograd method was $12 \% .{ }^{[8]}$ Likewise, in our study, 10 (14\%) Winograd group patients had a recurrence. Vandenbos ${ }^{[3]}$ reported the recurrence rate as $0 \%$ in his original article. Likewise, in the case series conducted using the Vandenbos procedure, the authors did not report recurrence in any patient. ${ }^{[4]}$ In the study, Noël ${ }^{[12]}$ recently described a procedure similar to the Vandenbos procedure, but performed different approximation sutures. In this study, 23 patients were under follow-up for one year, and no recurrence was observed in any of the patients. In the present study, we did not observe recurrence in any of our patients in the Vandenbos group.

It is important to achieve patient satisfaction and a satisfactory cosmetic result in the treatment of ingrown toenails. Although the patient is satisfied with the treatment, s/he may complain of the cosmetic appearance. In the study by Kose et al., ${ }^{[13]}$ the Winograd method was used for all patients and, although $97 \%$ of the patients were satisfied with the treatment of the disease, $8.8 \%$ of them complained in terms of cosmetics. The patients with complaints complained of inability to wear peep-toe shoes due to cosmetic reasons, such as narrowing and asymmetric shape of the nail and incision scar on the proximal part. The study conducted by Beytemur et al. ${ }^{[14]}$ found the dissatisfaction rate after the Winograd method as $12.1 \%$. In a case series performed using the Vandenbos procedure, almost all patients were satisfied with the procedure and in terms of cosmetics. ${ }^{[4]}$ In the present study, the Vandenbos procedure was found to be more satisfactory and cosmetically better than the Winograd method.

Recovery time after the surgical treatment of ingrown toenails is an important factor for patients. In the comparative study by Peyvandi et al., ${ }^{[8]}$ 
the return-to-work time was two weeks for the Winograd group. Likewise, in another study, the mean recovery time after the Winograd method was reported as two to four weeks. ${ }^{[10]}$ In their study, Chapeskie and $\mathrm{Kovac}^{[4]}$ found the recovery time as four to six weeks after the Vandenbos procedure, and our study showed it as 18 days. We consider that the recovery time is shorter in the study, as Chapeskie and $\mathrm{Kovac}^{[4]}$ considered the recovery time to return to normal activity as the recovery time. In our study, the time of wearing shoes was accepted as recovery time. Similar to the literature, the mean recovery time was 11 days for the Winograd group in our study, while it was 18 days for the Vandenbos group. We believe that this difference between the two groups is due to the longer duration of secondary recovery in the Vandenbos procedure.

When the VAS pain scores were compared between the two groups, the postoperative firstweek VAS scores were found to be higher in the patients who underwent the Vandenbos procedure, compared to those who were treated with the Winograd method. No clinical difference was observed between the VAS scores after one week. Although there is no study on postoperative pain scores for ingrown toenails in the literature, we attributed the higher one-week VAS scores after the Vandenbos procedure to more aggressive tissue excision.

In a study conducted by Huang et al., ${ }^{[15]}$ an infection rate of $3.2 \%$ was reported as a complication after the Winograd method for an ingrown toenail. In the current study, postoperative bleeding was observed in two patients in the Vandenbos procedure group, while the infection was observed in six patients, spicule formation was noted in three patients, and excessive granulation tissue was detected in two patients in the Winograd group. The comparison of the two groups showed that a higher number and variety of postoperative complications occurred in the Winograd group.

The limitations of the present study are that this is a retrospective study including a heterogeneous study population, and the patients were unable to be compared according to their ingrown toenail stages. Comparison of patients according to their stages can provide more detailed results. Due to the retrospective nature of the study, patients' satisfaction and cosmetic satisfaction were evaluated as Yes/No by reviewing the outpatient notes. More objective results would have been obtained, if satisfaction levels were able to be evaluated through a questionnaire.
In conclusion, the Winograd method can be preferred for patients who desire a rapid recovery owing to its shorter return-to-work time; however, the possibility of recurrence and complications should be kept in mind. The Vandenbos procedure has a low recurrence and higher cosmetic satisfaction rate. We consider that the appropriate surgical technique should be selected based on the individual patient. The difference between the two techniques can be demonstrated more clearly with large-scale, prospective, randomized studies.

\section{Declaration of conflicting interests}

The authors declared no conflicts of interest with respect to the authorship and/or publication of this article.

\section{Funding}

The authors received no financial support for the research and/or authorship of this article.

\section{REFERENCES}

1. André MS, Caucanas M, André J, Richert B. Treatment of ingrowing toenails with phenol $88 \%$ or trichloroacetic acid 100\%: A comparative, prospective, randomized, doubleblind study. Dermatol Surg 2018;44:645-50.

2. Borges APP, Pelafsky VPC, Miot LDB, Miot HA. Quality of life with ingrown toenails: A cross-sectional study. Dermatol Surg 2017;43:751-3.

3. Vandenbos $K Q$, Bowers WF. Ingrown toenail: a result of weight bearing on soft tissue. U S Armed Forces Med J 1959;10:1168-73.

4. Chapeskie H, Kovac JR. Case Series: Soft-tissue nail-fold excision: A definitive treatment for ingrown toenails. Can J Surg 2010;53:282-6.

5. Winograd AM. A modification in the technic of operation for ingrown toe-nail. 1929. J Am Podiatr Med Assoc 2007;97:274-7.

6. Bos AM, van Tilburg MW, van Sorge AA, Klinkenbijl $\mathrm{JH}$. Randomized clinical trial of surgical technique and local antibiotics for ingrowing toenail. Br J Surg 2007;94:292-6.

7. Gerritsma-Bleeker CL, Klaase JM, Geelkerken RH, Hermans J, van Det RJ. Partial matrix excision or segmental phenolization for ingrowing toenails. Arch Surg 2002;137:320-5.

8. Peyvandi H, Robati RM, Yegane RA, Hajinasrollah E, Toossi P, Peyvandi AA, et al. Comparison of two surgical methods (winograd and sleeve method) in the treatment of ingrown toenail. Dermatol Surg 2011;37:331-5.

9. Atik OS.. What are the expectations of an editor from a scientific article? Jt Dis Relat Surg 2020;31:597-8.

10. Vaccari S, Dika E, Balestri R, Rech G, Piraccini BM, Fanti PA. Partial excision of matrix and phenolic ablation for the treatment of ingrowing toenail: A 36-month followup of 197 treated patients. Dermatol Surg 2010;36:128893.

11. Kruijff S, van Det RJ, van der Meer GT, van den Berg IC, van der Palen J, Geelkerken RH. Partial matrix excision or orthonyxia for ingrowing toenails. J Am Coll Surg 2008;206:148-53. 
12. Noël B. Surgical treatment of ingrown toenail without matricectomy. Dermatol Surg 2008;34:79-83.

13. Kose O, Guler F, Gurcan S, Arik HO, Baz AB, Akalin S. Cosmetic results of wedge resection of nail matrix (Winograd technique) in the treatment of ingrown toenail. Foot Ankle Spec 2012;5:241-4.

14. Beytemur O, Dincel YM, Adanır O, Baris A, Alagoz E,
Pulatkan A. The results of the winograd method in surgical treatment of ingrown toenail. Istanbul Med J 2013; 14:227-30.

15. Huang JZ, Zhang YJ, Ma X, Wang X, Zhang C, Chen L. Comparison of wedge resection (Winograd procedure) and wedge resection plus complete nail plate avulsion in the treatment of ingrown toenails. J Foot Ankle Surg 2015;54:395-8. 